\title{
Treatment of Post-Transplant Recurrent FSGS in Children Using Plasmapheresis and Augmentation of Immunosuppression
}

\section{Jaime Restrepo-Tovar}

Fundación Valle del Lili

Laura Torres-Canchala ( $\square$ laura.torres@fvl.org.co)

Fundación Valle del Lili

Hernando Londoño

Fundación Valle del Lili

Michael Somers

Boston Children's Hospital

\section{Eliana Manzi}

Fundación Valle del Lili

Jaime Restrepo-Restrepo

Fundación Valle del Lili

\section{Research Article}

Keywords: Focal and segmental glomerulosclerosis, renal transplantation, recurrence, plasmapheresis, cyclosphosphamide, pediatric

Posted Date: January 18th, 2021

DOl: https://doi.org/10.21203/rs.3.rs-137756/v1

License: @ (i) This work is licensed under a Creative Commons Attribution 4.0 International License. Read Full License 


\section{Abstract}

Background: Up to $60 \%$ of pediatric renal transplant recipients with end-stage renal disease due to primary focal and segmental glomerulosclerosis (FSGS) may develop recurrent disease. Such recurrence is associated with poor prognosis if no remission is achieved. We report a single center experience with a protocol based on plasmaphereis and increased immunosuppression that resulted in a high long-lived remission rates.

Methods: This retrospective cohort study included consecutive pediatric renal transplant patients with recurrent FSGS treated with a standardized protocol using plasmapheresis and cyclophosphamide to supplement usual post-transplant immunosuppression with calcineurin inhibitors and steroids. Relapse was defined as urinary protein/creatinine ratio $>1.0 \mathrm{~g} / \mathrm{g}$ and remission as $<0.5 \mathrm{~g} / \mathrm{g}$.

Results: 15 patients with FSGS recurrence post-transplant were treated. All had therapy-resistant FSGS in native kidneys and had been on dialysis from 4-10 years. Of the 15, one died perioperatively from a pulmonary thromboembolism. 13 others achieved a complete remission within 3 months of treatment for FSGS recurrence. After a median follow-up period of 4 years, there were no recurrences of significant proteinuria.

Conclusion: The addition of plasmapheresis and cyclophosphamide to a calcineurin- and steroid-based immunosuppression regime was highy successful in inducing high remission rates with recurrent FSGS. Prospective trials are needed to evaluate further the efficacy of increased immunosuppression along with plasmapheresis in this setting.

\section{Introduction}

While renal transplantation is the treatment of choice for children with end-stage renal disease (ESRD)(1), some diseases such as primary focal and segmental glomerulosclerosis (FSGS) recur in the allograft and adversely affect graft outcomes if no remission is achieved $(2,5-7)$. Recurrence rate with FSGS as high as $60.5 \%$ have been reported, and concern for recurrence is widespread when considering renal transplantation in children with FSGS (4). Although a large number of therapies have been employed to treat FSGS recurrence, there is currently no consensus treatment $(2,3)$, and most of the treatments have not been evidence-based.

Historically, plasmapheresis (PLEX) is the most widely used treatment modality for recurrent FSGS in both children and adults $(2,6)$. One meta-analysis comprised of a single large series of 432 patients as well as several smaller case studies found a partial or complete remission rate of $71 \%$ with PLEX (8). There may have been substantial reporting bias in this cohort, however, and the number of PLEX treatments varied slgnificantly, with poor definition of other potentially beneficial concurrent therapies.

Similarly, the reports of PLEX efficacy in pediatric patients has varied. A recent publication in Pediatric Transplantation reported a limited impact of PLEX in treating FSGS recurrence (9), but on the other hand, 
a study of recurrent FSGS in Israeli children demonstrated a $78 \%$ partial or complete response, similar to the meta-analysis results (10). There has also been interest coupling PLEX with Rituxumab or using LDLapheresis rather than conventional plasma exchange, though reports of these approaches have also been limited $(11,12,13)$.

Another approach for the treatment of FSGS has focused on increased immunosuppression along with PLEX (7). Nathanson reported that $6 / 14$ patients with FSGS recurrence achieved complete remission by using a combination of high dose cyclosporine, PLEX, and oral cyclophosphamide (7). To discern if there were similar efficacy, we were interested in evaluating our experience with a similar protocol that adds cyclophosphamide to calcineurin inhibitors, steroids, and PLEX for post-transplant FSGS recurrence in children and adolescents.

\section{Methods}

\section{Setting and Study Population}

This study was performed at Fundación Valle del Líli Hospital in Cali, Colombia, a tertiary care university institution. Our long-standing pediatric renal transplant program keeps a local registry of all renal transplant patients at the institution. In our retrospective analysis, we included all patients $<18$ years of age transplanted between 1998-2015 due to primary FSGS who manifested FSGS recurrence posttransplant and were treated with a protocol for FSGS recurrence based on the use of PLEX and addition of cyclophosphamide to baseline immunosuppression with a calcineurin inhibitor and steroids. This study was performed in accordance with the Declaration of Helsinki and was approved by the Fundación Valle del Lili ethics board committee. Informed written consent was waived by the Fundación Valle del Lili ethics board committee ("Comité de ética en Investigación Biomédica" in Spanish) since it was a retrospective cohort study without influencing patient care.

\section{Definitions, Anthropometrics, and Laboratory Measurements}

Recurrence was defined as a serum albumin level below $3 \mathrm{~g} / \mathrm{dL}$, either in the setting of a urinary protein/creatinine ratio (UPC) $>1 \mathrm{~g} / \mathrm{g}$ presenting in the first 24 to 72 hours after transplantation or the later onset of UPC $>1 \mathrm{~g} / \mathrm{g}$ post-transplant distinct from other events such as acute rejection, acute kidney injury, or chronic allograft nephropathy, all documented by biopsy. Remission was defined as UPC < $0.5 \mathrm{~g} / \mathrm{g}$ and serum albumin level $>3 \mathrm{~g} / \mathrm{dL}$.

Height was measured without shoes on a wall mounted stadiometer and weight was measured without heavy clothes using either a digital or balance-beam scale. Body mass index (BMI) was calculated as weight $(\mathrm{kg}) /$ height $\left(\mathrm{m}^{2}\right)$. We calculated age- and gender- independent height and weight z-scores as previously described, using the Ped(z) app with the WHO reference intervals.(14) 
Blood samples were collected in anticoagulant and lithium heparin tubes. Creatinine was isotope dilutionmass spectrometry traceable and measured using a calorimetric modified Jaffe method and alkaline picrate.(15) Estimated glomerular filtration rate (eGFR) was calculated from the average of the three height measurements and serum creatinine with the new modified Schwartz formula.(16)

\section{Peri- and Post-Transplant Management}

(a) All living donor (LD) recipients received 3 pre-emptive PLEX treatments during the week prior to transplantation and IV cyclosporine at $3.5 \mathrm{mg} / \mathrm{Kg} /$ day over the 24 hours prior to transplant.

(b) Induction therapy was chosen at the discretion of the transplant surgeon or the transplant nephrologist and varied by era of transplantation. Five patients (33\%) received thymoglobulin at a dose of $1-1.5 \mathrm{mg} / \mathrm{kg} /$ dose for a total of 4 daily doses maximum. Ten patients $(67 \%)$ received a monoclonal anti IL-2 receptor antibody (basiliximab or daclizumab). Basiliximab was given at a dose of $20 \mathrm{mg}$ in children $<20 \mathrm{Kg}(\mathrm{n}=8[80 \%])$ or $30 \mathrm{mg}$ in larger children, with the first dose on the day of transplant and the second dose on day 4. Daclizumab $(n=2[20 \%])$ was provded at a dose of $1 \mathrm{mg} / \mathrm{kg}$ on the day of transplant and a second similar dose 2 weeks later.

(c) Steroid therapy: IV Prednisolone $(10 \mathrm{mg} / \mathrm{kg}$ ) daily for 3 days followed by oral prednisone tapering ( $1 \mathrm{mg} / \mathrm{kg} /$ day) over 2 months to a maintenance dose of $0.2 \mathrm{mg} / \mathrm{kg} /$ day for two years. Steroids were then tapered to alternate day for one more year and then discontinued.

(d) Calcineurin inhibitor therapy: High dose microemulsified cyclosporine A was prescribed at 8$10 \mathrm{mg} / \mathrm{kg} /$ day every 12 hours, targeting a C2 level of $1200-1500 \mathrm{mcg} / \mathrm{L}$, for 3 months. If the patients had no recurrence, they were then transitioned from cyclosporine to tacrolimus at a dose of $0.1-$ $0.2 \mathrm{mg} / \mathrm{kg} /$ day. Trough target levels of tacrolimus were $8-10 \mu \mathrm{g} / \mathrm{L}$ for 6 months and then $6-8 \mu \mathrm{g} / \mathrm{L}$.

(e) Mycophenolate mofetil (MMF) at 600-900 mg/m²/day. No MMF monitoring was employed.

(f) Cytomegalovirus Prophylaxis: Since 2008, all patients received daily oral valganciclovir prophylaxis $\left(7 \mathrm{mg} / \mathrm{m}^{2} \mathrm{x}\right.$ creatinine clearance(16)) for cytomegalovirus prophylaxis for a total of 6 months.

\section{Treatment Protocol for FSGS Recurrence}

(g) Upon diagnosis of recurrence, PLEX was initiated with 4 daily sessions, followed by every other day sessions as long as UPC $>0.5$. If after 15 PLEX sessions there was no significant improvement in proteinuria, PLEX was discontinued. PLEX treatments consisted of 1.5 plasma volume exchange swith $5 \%$ albumin as replacement fluid through a central vascular access using a Cobe Spectra System.

(h) Cyclophosphamide: Oral cyclophosphamide was prescribed at $2 \mathrm{mg} / \mathrm{kg} /$ day daily once daily for 3 months in all patients who had FSGS recurrence, regardless of the cumulative dose of cyclophosphamide that may have been provided prior to transplantation to treat native FSGS. When cyclophosphamide was started, MMF was held. When cyclophosphamide was completed, MMF was re-started at $600-$ $900 \mathrm{mg} / \mathrm{m}^{2} /$ day. 
(i) Calcineurin inhibitors: As detailed above, microemulsified cyclosporine A at a dose of $8-10 \mathrm{mg} / \mathrm{kg} / \mathrm{day}$ in two divided doses was provided, targeting a C2 level of $1200-1500 \mathrm{mcg} / \mathrm{L}$, for 3 If remission were achieved, patients were then converted after 3 months to tacrolimus at a dose of $0.1-0.2 \mathrm{mg} / \mathrm{kg} / \mathrm{day}$, targeting through levels of $8-10 \mu \mathrm{g} / \mathrm{L}$ during the first year, and then $6-8 \mu \mathrm{g} / \mathrm{L}$ thereafter.

(j) Rituximab: In one child who had persistent leukopenia during cyclophosphamide therapy, a single dose of rituximab $375 \mathrm{mg} / \mathrm{m}^{2}$ was provided when the cyclophosphamide therapy needed to be stopped prior to its full provision. In a second patient with partial remission after PLEX and completion of cyclophosphamide, rituximab was also provided, with four weekly doses of $375 \mathrm{mg} / \mathrm{m}^{2}$.

\section{Statistical methods}

Anthropometric z-scores were calculated using the app Ped(z) based on WHO growth charts.(14) We used simple descriptive statistics wherever possible. Continuous variables were analyzed for normal distribution using the D'Agostino-Pearson normality test.(17) No adjustments were made for missing data. All calculations were performed using GraphPad Prism version 5.0f for Mac or STATA 14.0 for PC registered to LTC.

\section{Results}

17 children with end-stage renal failure due to primary FSGS received their first kidney transplant at Fundación Valle del Lilí and were part of the local transplant regisrty. Fifteen (88\%) experienced recurrence of FSGS and were treated according ot the protocol outlined above. Table 1 presents demographic and clinical management characteristics of transplanted children with recurrence.

Nine patients (60\%) were boys. The median age at diagnosis of primary FSGS was 8 years (IQR 6.110.3). None of the patients was HIV positive, diabetic, or obese, or had any other cause of secondary FSGS. Genetic studies were not performed prior to transplantation in any child.

Prior to ESRD diagnosis, 14 of the 15 patients manifested steroid-resistance, with one child showing partial responsiveness. Prior to ESRD, all children had also been treated with non-steroid immunosuppression tryng to induce FSGS remission, including 7 who received cyclosporine, 6 who received cyclophosphamide, 3 who received MMF, and 2 who received Rituximab. 
Table 1

Demographics and Characteristics of Recurrent FSGS Patients

\begin{tabular}{|ll|}
\hline Characteristics & $\mathbf{N}=15$ \\
\hline Male, $\mathbf{n}(\%)$ & $9(60.0)$ \\
\hline Age at transplant, years. Median (IQR) & $14.3(9.5-17.6)$ \\
\hline Time between diagnosis and transplant, years. Median (IQR) & $6.5(3.1-7.7)$ \\
\hline Donor type, $\mathbf{n}(\%)$ & \\
\hline Live donor & $8(53.3)$ \\
\hline Deceased donor & $7(46.7)$ \\
\hline Anthropometrical measurements, & \\
\hline Weight [Kg], median (IQR) & $32(23-48)$ \\
\hline Weight z-score, mean ( \pm SD) & $-1.3(1.2)$ \\
\hline Height [cm], median (IQR) & $148(123-156)$ \\
\hline Height z-score, mean ( \pm SD) & $-1.8(0.96)$ \\
\hline BMI [Kg/m2], median (IQR) & $18.3(15.2-19.3)$ \\
\hline BMI z-score, mean ( \pm SD) & $-0.7(1.1)$ \\
\hline Previous nephrectomy, $\mathrm{n}(\%)$ & $10(66.7)$ \\
\hline Pre-transplant dialysis modality, $\mathbf{n}(\%)$ & $23(17-43)$ \\
\hline Hemodialysis & $8(53.3)$ \\
\hline Peritoneal dialysis, & $7(46.7)$ \\
\hline Induction therapy, $\mathbf{n}(\%)$ & $5(33.3)$ \\
\hline Thymoglobulin & $7(46.7)$ \\
\hline Basiliximab & \\
\hline Daclizumab & \\
\hline Post-Trasnplant Plasmapheresis Procedure & \\
\hline Median Number (IQR) & \\
\hline Length of hospital stay (days), median (IQR) & \\
\hline
\end{tabular}

All children reached ESRD and required dialysis prior to transplantation (range 4-10 years). Ten patients (58\%) required nephrectomy prior to transplant due to ongoing nephrosis or severe proteinuria. The median age at transplant was 14.3 years (IQR 3.1-7.7). Eight patients (53\%) had a living donor graft and 
$7(47 \%)$ a deceased donor graft. There were no differences in patient characteristics between children who received living related and deceased donor transplants.

In the 15 patients with recurrent FSGS who received post-transplant PLEX and augmented immunosuppression per protoccol, the median number of PLEX procedures provided was 8 (IQR 5-10, range 4 to 18). Complete remission was achieved by 12 patients after starting PLEX and augmenting immunosuppression, with an additional patient achieved remission with the addition of rituximab after not responding to the course of cyclophosphamide and PLEX. One patient died of a pulmonary thromboembolism within 2 weeks of transplant while receiving PLEX therapy for recurrence in a stillfunctioning graft.

Table 2 details quantification of proteinuria longitudinally and Figure 1 shows proteinuria remission over time. All patients who achieved remission stayed in remission without further disease recurrence during follow-up (median 4 years; IQR 2.2-7.8 years). In the single patient who did not remit, proteinuria transiently exacerbated further about three months after transplant concomitant with medication nonadherence, but then returned to prior levels.

Table 2

Longitudinal Proteinuria

\begin{tabular}{|ll|}
\hline Protein/Creatinine ratio, median (IQR) & $\mathbf{n = 1 5}$ \\
\hline At diagnosis of recurrence & $6.2(3.0-10.1)$ \\
\hline At hospital discharge & $0.35(0.25-0.66)$ \\
\hline 3 months post-transplant & $0.3(0.25-0.4)$ \\
\hline 12 months post-transplant & $0.095(0.08-0.10)$ \\
\hline 72 months post-transplant & $0.1(0.095-0.16)$ \\
\hline
\end{tabular}

Table 3 details graft function by estimated GFR. Median estimated GFR stayed stable during the first year after transplant. At last follow-up at a median of 4 years after transplant, there had been a median decline of eGFR by $15 \mathrm{ml} / \mathrm{min} / 1.73 \mathrm{M}^{2}$

In the child with persistent nephrosis, graft function was lost 64 months after transplant. Three other grafts were also lost during long-term follow-up: one related to inability to obtain immunosuppression and the others related to chronic allograft nephropathy. None of these patients had significant proteinuria at the time of graft loss. All patients who lost grafts subsequently died while on dialysis, two of infection, one from sequelae of a hypertensive crisis, and one as a young adult of colon cancer after having had another kidney transplant that also failed due to FSGS recurrence. 
Table 3

Longitudinal Renal Function

\begin{tabular}{|c|c|}
\hline Renal function & $n=15$ \\
\hline \multicolumn{2}{|l|}{ At 7 days, median (IQR) } \\
\hline Serum creatinine $[\mathrm{mg} / \mathrm{dL}]$ & $1.63(0.58-2.89)$ \\
\hline Schwartz eGFR $\left[\mathrm{mL} / \mathrm{min} / 1.73 \mathrm{~m}^{2}\right]$ & $50.6(27.5-84.6)$ \\
\hline \multicolumn{2}{|l|}{ At 15 days, median (IQR) } \\
\hline Serum creatinine $[\mathrm{mg} / \mathrm{dL}]$ & $1.1(0.7-2.8)$ \\
\hline Schwartz eGFR $\left[\mathrm{mL} / \mathrm{min} / 1.73 \mathrm{~m}^{2}\right]$ & $61.2(27.1-78.8)$ \\
\hline \multicolumn{2}{|l|}{ At 30 days, median (IQR) } \\
\hline Serum creatinine $[\mathrm{mg} / \mathrm{dL}]$ & $1.0(0.8-2.9)$ \\
\hline Schwartz eGFR $\left[\mathrm{mL} / \mathrm{min} / 1.73 \mathrm{~m}^{2}\right]$ & $60.3(28.6-67.9)$ \\
\hline \multicolumn{2}{|l|}{ At discharge, median (IQR) } \\
\hline Serum creatinine $[\mathrm{mg} / \mathrm{dL}]$ & $1.0(0.8-1.7)$ \\
\hline Schwartz eGFR $\left[\mathrm{mL} / \mathrm{min} / 1.73 \mathrm{~m}^{2}\right]$ & $61.0(42.2-74.0)$ \\
\hline \multicolumn{2}{|l|}{ At year, median (IQR) } \\
\hline Serum creatinine $[\mathrm{mg} / \mathrm{dL}]$, & $1.04(0.78-2.3)$ \\
\hline Schwartz eGFR $\left[\mathrm{mL} / \mathrm{min} / 1.73 \mathrm{~m}^{2}\right]$ & $57.7(30.7-77.1)$ \\
\hline \multicolumn{2}{|l|}{ At last follow-up, median (IQR) } \\
\hline Time of follow-up & $4.0(2.2-7.8)$ \\
\hline Serum creatinine $[\mathrm{mg} / \mathrm{dL}]$ & $1.63(1.2-2.3)$ \\
\hline Schwartz eGFR [mL/min/1.73 m²] & $42.8(33.1-55.8)$ \\
\hline \multicolumn{2}{|l|}{ Protein/Creatinine ratio, median (IQR) } \\
\hline At diagnosis of recurrence & $6.2(3.0-10.1)$ \\
\hline At hospital discharge & $0.35(0.25-0.66)$ \\
\hline 3 months post-transplant & $0.3(0.25-0.4)$ \\
\hline 12 months post-transplant & $0.095(0.08-0.10)$ \\
\hline 72 months post-transplant & $0.1(0.095-0.16)$ \\
\hline
\end{tabular}




\section{Discussion}

In this single center retrospective review of 17 children transplanted due to FSGS, 15 or $88 \%$ had recurrent disease and were treated for their recurrence with a protocol using PLEX and augmentation of immunosuppression. Of the children with recurrence, 13 or $87 \%$ were able to enter into a long-lived complete remission. In all but one case, the remissions occurred quickly after insitituion of therapy for recurrence, and only one child required supplemental therapy with the addition of rituximab after not fully remitting after PLEX and cyclophosphamide provision.

Although a large meta-analysis of patients with FSGS recurrence demonstrated a similar $71 \%$ rate of response with PLEX-based therapies, nearly $30 \%$ achieved only a partial remission, and the number of PLEX sessions and the duration of PLEX therapy was often substantial. In comparison, the children described in our report tended to have rapid and complete responses, and there were no subsequent relapses during long-term follow up. Moreover, eGFR remained acceptable long-term, with eGFR decline over time similar to rates seen more generally in children.

There is currently no consensus about the best overall approach for the treatment of recurrent FSGS in children $(2,4)$, largely due to the lack of prospective randomized controlled trials. Moreover, although PLEX is quite often used as a first-line therapy for recurrence, it is almost always provided in the setting of other additional therapies, and there is little data to guide clinicians as to the specific efficacy of individual components of such multi-tiered management (19).

The decision to augment immunosuppression in FSGS recurrence in children is further complicated since most of these children have already had significant pre-transplant exposure to immunosuppression as part of attempts to reverse FSGS in their native kidneys. Concerns for cumulative toxicity of certain medications, such as cyclophosphamide, may lead to decisions to forgo the use of what may be an efficacious therapy, especially when there is not clarity as to the best overall therapy or the best population for that drug's efficacy.

The paucity of prospective controlled studies not only exacerbates the lack of clarity as to best current management strategies but also complicates the assessment of novel therapies as they become available. For instance, rituximab and LDL-apheresis have both been used increasingly to treat FSGS recurrence, but their utility in children with recurrent disease in general is difficult to assess when there has been little formal assessment of current therapies to allow baseline levels of expectation.

The importance of identifying the best therapies is underscored by long-term outcomes in successfully transplanted patients as compared to those with failed allografts. FSGS recurrence that does not respond to treatment leads to early graft loss and return to dialysis, and most often protends future episodes of recurrence with subsequent transplantation. The long-term reliance on dialysis for renal replacement therapy adversely affects quality of life, requires more health care resources and is more costly, and results in significantly shorter life expectancy. In our own cohort, despite the successful treatment of 
recurrence in almost all patients, those who went on to lose graft function for other reasons and then required chronic dialysis as young adults all had limited life spans.

Although the conclusions that can be drawn from our results may not be generalizable to all children with recurrent FSGS, our report does show that in some children an aggressive approach to recurrence that uses PLEX and immunosuppression augmentation including cyclophosphamide can lead to rapid, complete, long-term remission. Rigorous assessment of proposed protocols and their effectiveness in children with FSGS should help to determine what therapies need to be studied in a randomized, controlled fashion in children and underscore the importance of sharing results such as described with this reported cohort to the pediatric nephrology community at large.

\section{Declarations}

\section{Ethics approval and consent to participate.}

This study was in accordance with the Declaration of Helsinki and was approved by the Fundación Valle del Lilí ethics board committee (Comité de Ética en Investigación Biomédica). Informed written consent was waived by the local ethics board since it was a retrospective cohort study without influencing patient care.

\section{Consent for publication}

As this was a retrospective study without impact on patient care, the need for written informed consent was waived by the research ethics board.

\section{Availability of data and materials}

The datasets generated and/or analysed during the current study are not publicly available in order to protect patient information in accordance with Declaration of Helsinki but are available from the corresponding author on reasonable request.

\section{Competing interests}

All the authors declare no competing interest related to this research.

\section{Funding:}

This study was supported by the International Society of Nephrology (ISN) through the ISN Sister Renal Center Program: Boston Children's Hospital USA - Fundación Valle del Lili, Cali, Colombia.

\section{Authors' contributions}

JAR and JMR conceived the study, designed the study and obtained ethics approval. JMR was involved in all aspects of the paper generation and revised each draft, contributed to the statistical analysis, and 
coordinated all coauthors' activities. JAR, HL, and EM collected all the data. LTC performed the statistical analysis. MJGS, LTC and JMR wrote the different versions. LTC also prepared the tables and figures. All authors interpreted the data. All authors provided critical input into all aspects of the design and execution of the study and participated in all phases of the paper writing. All authors participated in revising the manuscript critically for important intellectual content and approved the final version to be submitted to the journal.

\section{Acknowledgements:}

We thank the Centro de investigaciones clinicas of Fundación Valle del Lili for the strong support given during the entire research process. Dra. Laura Torres-Canchala was supported by the International Society of Nephrology for a two months research sabbatical at the University of Western Ontario.

\section{References}

1. Filler G. Challenges in pediatric transplantation: the impact of chronic kidney disease and cardiovascular risk factors on long-term outcomes and recommended management strategies. Pediatr Transplant 2011: 15: 25-31.

2. Francis A, Didsbury M, McCarthy $H$, Kara $T$. Treatment of recurrent focal segmental glomerulosclerosis post kidney transplantation in Australian and New Zealand children: a retrospective cohort study. Pediatr Transplant 2018: 22.

3. Filler G, Restrepo JM. The urgent need for more research on how to treat recurrent focal and segmental glomerulosclerosis. Pediatr Transplant 2018: e13215.

4. Cormican S, Kennedy C, O'Kelly P, et al. Renal transplant outcomes in primary FSGS compared with other recipients and risk factors for recurrence: A national review of the Irish Transplant Registry. Clinical transplantation 2018: 32.

5. Baum MA, Ho M, Stablein D, Alexander SR, North American Pediatric Renal Transplant Cooperative S. Outcome of renal transplantation in adolescents with focal segmental glomerulosclerosis. Pediatr Transplant 2002: 6: 488-492.

6. Runowski D, Prokurat S, Rubik J, Grenda R. Therapeutic Plasma Exchange in Pediatric Renal Transplantation Experience of One Decade and 389 Sessions. Transplant Proc 2018: 50: 34833486.

7. Nathanson S, Cochat $P$, Andre JL, et al. Recurrence of nephrotic syndrome after renal transplantation: influence of increased immunosuppression. Pediatr Nephrol 2005: 20: 1801-1804.

8. Kashgary A, Sontrop JM, Li L, et al. The role of plasma exchange in treating post-transplant focal segmental glomerulosclerosis: A systematic review and meta-analysis of 77 case-reports and caseseries. BMC nephrology 2016: 17: 104.

9. Verghese PS, Rheault MN, Jackson S, Matas AJ, Chinnakotla S, Chavers B. The effect of peritransplant plasmapheresis in the prevention of recurrent FSGS. Pediatr Transplant 2018. 
10. Cleper R, Krause I, Bar Nathan N, et al. Focal segmental glomerulosclerosis in pediatric kidney transplantation: 30 years' experience. Clinical transplantation 2016: 30: 1324-1331.

11. Shah L, Hooper DK, Okamura D, et al. LDL-apheresis-induced remission of focal segmental glomerulosclerosis recurrence in pediatric renal transplant recipients. Pediatr Nephrol 2019: 34: 2343-2350.

12. Raina R, Krishnappa V. An update on LDL apheresis for nephrotic syndrome. Pediatr Nephrol 2019: 34: $1655-1669$.

13. Filler G, Lee M, Hegele RA. Barriers to the Implementation of Lipoprotein Apheresis in Canada. Can J Cardiol 2017: 33: 409-411.

14. de Onis M, Onyango A, Borghi E, Siyam A, Blossner M, Lutter C. Worldwide implementation of the WHO Child Growth Standards. Public health nutrition 2012: 1-8.

15. Matas AJ, Smith JM, Skeans MA, et al. OPTN/SRTR 2012 Annual Data Report: kidney. Am J Transplant 2014: 14 Suppl 1: 11-44.

16. Schwartz GJ, Schneider MF, Maier PS, et al. Improved equations estimating GFR in children with chronic kidney disease using an immunonephelometric determination of cystatin C. Kidney Int 2012: 82: $445-453$.

17. RB DA. Tests for Normal Distribution: Macel Decker, 1986.

18. Freundlich M, Lipshultz SE, Filler G. Chronic kidney disease and cardiac morbidity - What are the possible links? Progress in Pediatric Cardiology 2016: 41: 89-95.

19. Kang HG, Ha IS, Cheong HI. Recurrence and Treatment after Renal Transplantation in Children with FSGS. BioMed research international 2016: 2016: 6832971.

20. Bacchetta J, Cochat P. Primary disease recurrence-effects on paediatric renal transplantation outcomes. Nature reviews Nephrology 2015: 11: 371-384.

21. Trachtman R, Sran SS, Trachtman H. Recurrent focal segmental glomerulosclerosis after kidney transplantation. Pediatr Nephrol 2015: 30: 1793-1802.

22. Francis A, Didsbury M, McCarthy $\mathrm{H}$, Kara $\mathrm{T}$. Treatment of recurrent focal segmental glomerulosclerosis post-kidney transplantation in Australian and New Zealand children: A retrospective cohort study. Pediatr Transplant 2018: 22: e13185.

\section{Figures}




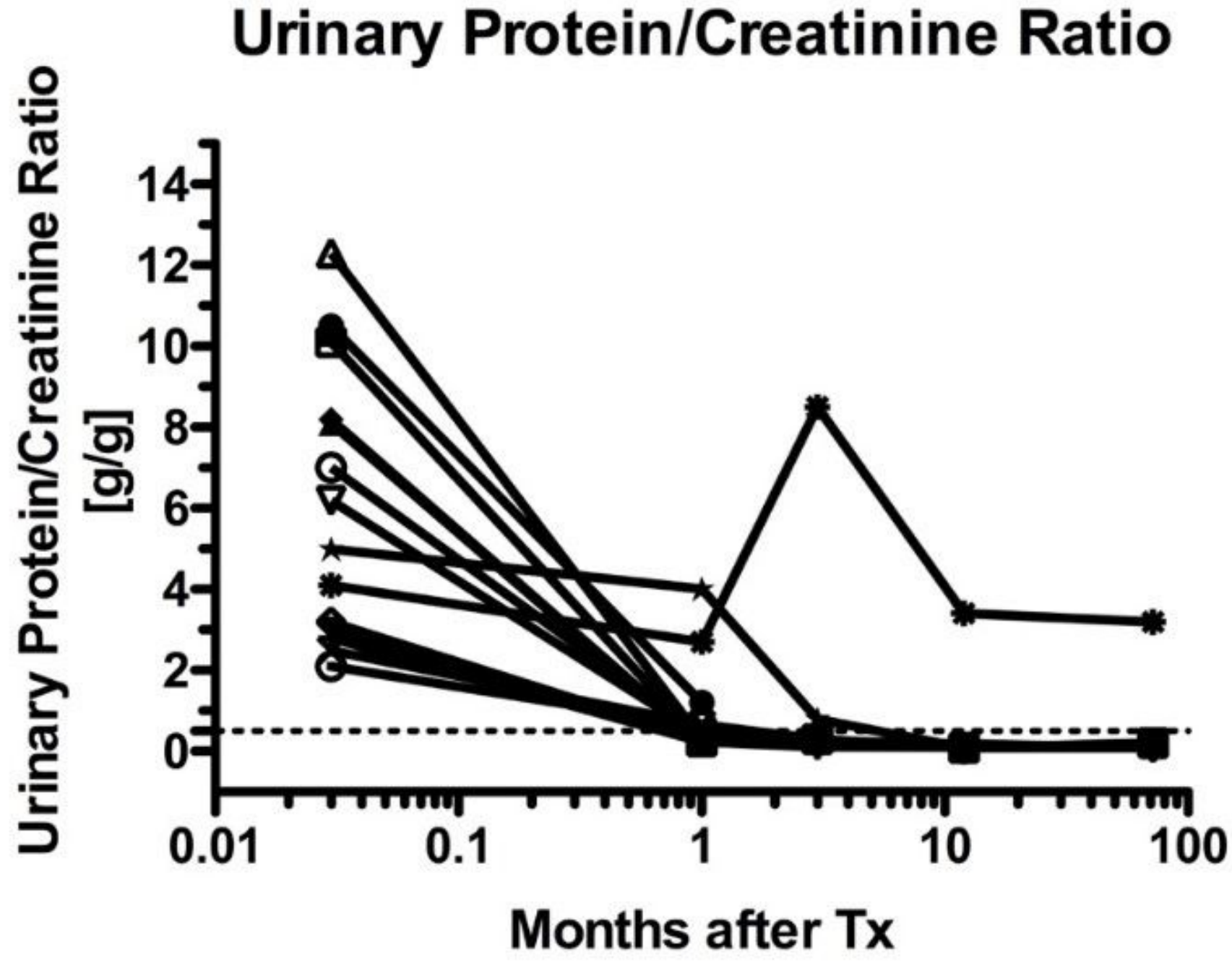

Figure 1

Post-transplant Remission from Proteinuria 